\title{
INEQUALITIES FOR THE ZEROS OF LEGENDRE POLYNOMIALS AND RELATED FUNCTIONS*
}

\author{
BY \\ GABRIEL SZEGÖ \\ INTRODUCTION
}

In what follows we deal mainly with some inequalities for the zeros of Legendre polynomials $P_{n}(\cos \theta)$, given by Bruns [1], $\dagger$ and later independently by $A$. Markoff [6] and by Stieltjes [8], improving the results of Bruns. Let $\theta_{1}, \theta_{2}, \cdots, \theta_{n}$ denote the zeros of $P_{n}(\cos \theta)$ in the interval $(0, \pi)$ in increasing order, so that

$$
0<\theta_{1}<\theta_{2}<\cdots<\theta_{n}<\pi .
$$

Then the inequalities of Bruns can be formulated as follows:

$$
\frac{\nu-\frac{1}{2}}{n+\frac{1}{2}} \pi<\theta_{\nu}<\frac{\nu}{n+\frac{1}{2}} \pi \quad(\nu=1,2, \cdots, n) .
$$

The improved inequalities due both to A. Markoff and Stieltjes are

$$
\frac{\nu-\frac{1}{2}}{n} \pi<\theta_{\nu}<\frac{\nu}{n+1} \pi \quad(\nu=1,2, \cdots \quad[n / 2]) .
$$

This concerns only the group of zeros lying in the interval $0<\theta<\pi / 2$. The symmetric property

$$
\theta_{\nu}+\theta_{n+1-\nu}=\pi
$$

yields however a similar estimate for the second group of zeros in the interval $\pi / 2<\theta<\pi$.

These inequalities indicate in particular the "regular distribution" of the systems of zeros in the interval $(0, \pi)$ if $n \rightarrow \infty$.

We show in the first part of this paper how the inequalities (2), (3) can be derived in a very simple way using the classical ideas of Sturm and the well known differential equation satisfied by the function $P_{n}(\cos \theta)$. The second part contains some elementary facts about the zeros of a class of trigonometric polynomials and gives also some inequalities for the zeros of $P_{n}(\cos \theta)$ derived on the basis of these facts.

* Presented to the Society, September 13, 1935; received by the editors February 2, 1935.

$\dagger$ Numbers in bold face type refer to the Bibliography at the end of this paper. 
We start in $\S I$ with a formulation of a theorem of Sturm adapted to our later needs. In §II we prove (2) and (3) by means of Sturm's method obtaining the lower estimate of (3) in even a sharper form, $\left[\left(\nu-\frac{1}{4}\right) \pi /\left(n+\frac{1}{2}\right)\right.$ instead of $\left.\left(\nu-\frac{1}{2}\right) \pi / n\right]$. The proof of the upper bound is based on the following remarkable property of the zeros in question, which is a simple consequence of Sturm's theorem. The sequence

$$
0=\theta_{0}, \theta_{1}, \theta_{2}, \cdots, \theta_{\rho+1}, \quad \rho=[n / 2],
$$

is convex, that is to say, the differences $\theta_{\nu}-\theta_{\nu-1}$ are increasing if $\nu$ runs from 1 to $\rho+1$. [Cf. the hint in Hille 5, p. 162.]

§III treats of some analogous properties of the Bessel function $J_{0}(\theta)$ by Sturm's method. We obtain some new inequalities for the zeros $\theta_{\nu}$ in terms of the zeros of $J_{0}(\theta)$. There is no difficulty in extending these results to some ultra-spherical polynomials and to general Bessel functions ( $\$ I V)$.

In the second part we first consider trigonometric cosine polynomials

$$
\lambda_{0} \cos m t+\lambda_{1} \cos (m-1) t+\cdots+\lambda_{m-1} \cos t+\lambda_{m}
$$

with positive and monotonically increasing coefficients:

$$
\lambda_{0}>\lambda_{1}>\lambda_{2}>\cdots>\lambda_{m}>0 .
$$

Pólya has shown $[7$, p. 359], by a simple application of the principle of the argument, that the zeros of such a polynomial are all real and simple. We prove that, under the condition mentioned (and even if equality holds in place of all inequalities (7) except the first), every interval

$$
\frac{\mu-\frac{1}{2}}{m+\frac{1}{2}} \pi<t<\frac{\mu+\frac{1}{2}}{m+\frac{1}{2}} \pi \quad(\mu=12, \cdots, m)
$$

contains exactly one of these zeros $(\S \mathrm{V})$. These inequalities yield, under a rather general condition, the "regular distribution" of the zeros for large values of $m$. The extremely simple proof is based on the classical fact that the sums

$$
\sin \frac{1}{2} t+\sin \frac{3}{2} t+\cdots+\sin \left(m+\frac{1}{2}\right) t \quad(m=0,1,2,3, \cdots)
$$

are positive in the interval $0<t<2 \pi$. As an application of our result we derive very simply the main theorem of Pólya's paper quoted above $(\S \mathrm{VI})$.

Legendre polynomials $P_{n}(\cos \theta)$ are not exactly of the form (6), (7). Our method gives however also in this case some inequalities for the zeros which are only a little less precise than those of Bruns. The same method can be applied to some generalizations of Legendre polynomials due to Fejér [3]. In special cases an improvement of these results can be easily obtained. We prove for instance the lower estimate in (3) by this elementary method. 


\section{Part 1. Applications of Sturm's method}

\section{Preliminaries}

1. With regard to the later applications it is advantageous to formulate Sturm's theorem in the following form:

Let $f(x)$ and $F(x)$ be continuous functions in $a<x \leqq b$ and let there $f(x) \leqq F(x)$ but $f(x) \not \equiv F(x)$. Let the functions $y(x)$ and $Y(x)$ satisfy in $a<x \leqq b^{*}$ the differential equations

$$
y^{\prime \prime}+f(x) y=0, \quad Y^{\prime \prime}+F(x) Y=0,
$$

and further the following conditions:

$$
\begin{gathered}
y(x)>0 \text { in } a<x<b, \quad y(b)=0 ; \\
\lim _{x \rightarrow a+0}\left\{y^{\prime}(x) Y(x)-y(x) Y^{\prime}(x)\right\} \text { exists and } \geqq 0 .
\end{gathered}
$$

Then either the function $Y(x)$ is identically zero or it assumes negative values in some subintervals of $(a, b)$.

It may be observed that our equations are not necessarily satisfied for $x=a$.

The essential idea of the proof is well known. Namely as a consequence of the assumption $Y(x) \geqq 0, Y(x) \not \equiv 0$ in $a<x<b$, we have

$$
\left(y^{\prime} Y-y Y^{\prime}\right)_{\substack{x=b \\ x=x_{1}}}^{\substack{b=\\ x_{1}}}(F(x)-f(x)) Y(x) y(x) d x \geqq K>0,
$$

provided that $a<x_{1}<b$ and $x_{1}-a$ is sufficiently small. Here the positive number $K$ is independent of $x_{1}$. Consequently we have [cf. (3)]

$$
y^{\prime}(b) Y(b)-y(b) Y^{\prime}(b)=y^{\prime}(b) Y(b)>0 .
$$

Now, by $(2), y^{\prime}(b)<0$, whence $Y(b)<0$, which is a contradiction.

If the limit in (3) is $\leqq 0$, the statement is changed in an obvious way. If in addition to (2) the condition

$$
\lim _{x \rightarrow a+0}\left\{y^{\prime}(x) Y(x)-y(x) Y^{\prime}(x)\right\}=0
$$

is satisfied, $Y(x)$ is either identically zero or it has at least one variation of sign in $a<x<b$.

The same statement holds in the well known classical case in which conditions (2) and $\left(3^{\prime}\right)$ are replaced by

${ }^{*}$ For $x=b$ this means that the left-hand derivatives of the first and second order exist at $x=b$ and satisfy differential equations (1). We write for brevity $y^{\prime}(b-0)=y^{\prime}(b), y^{\prime \prime}(b-0)=y^{\prime \prime}(b)$ and so on. 


$$
y(x)>0 \text { in } a<x<b, y(a)=y(b)=0,
$$

and both differential equations (1) are satisfied in the closed interval $a \leqq x \leqq b$. In this case $Y(x) \geqq 0, Y(x) \not \equiv 0, a<x<b$, implies

$$
y^{\prime}(b) Y(b)-y^{\prime}(a) Y(a)>0 .
$$

This is a contradiction because

$$
y^{\prime}(a)>0, \quad y^{\prime}(b)<0, \quad Y(a) \geqq 0, Y(b) \geqq 0 .
$$

2. We mention in this connection the following important application:

Let $\phi(x)$ be continuous and decreasing in $x_{0}<x<X_{0}$, and let $y$ be a solution of

$$
y^{\prime \prime}+\phi(x) y=0
$$

which is not identically zero. The sequence of zeros of $y$ is always convex, i.e., the sequence of differences of consecutive zeros is increasing.

This theorem also goes back to Sturm [9, p. 173; cf. also Hille 5]; it can be deduced by means of the following simple argument. Let $p<q<r<s<\ldots$. be the zeros in question, ${ }^{*} q-p=h$. We apply Sturm's theorem in the interval $(q, r)$ to the equations

$$
y^{\prime \prime}+\phi(x) y=0, Y^{\prime \prime}+\phi(x-h) Y=0,
$$

the second having the solution $Y(x)=y(x-h)$; it is evident that $\phi(x)<\phi(x-h)$, so that $r-q>h$, i.e., $r-q>q-p$.

Remarks. This proof, and consequently the last inequality, remain valid under the following more general assumption:

$$
\phi(x)>\phi(q) \text { for } x<q \text { and } \phi(x)<\phi(q) \text { for } x>q .
$$

Furthermore we can also have $p=x_{0}$ in the sense that $\lim _{x \rightarrow x_{0}+0} y(x)=0$, provided that condition $\left(3^{\prime}\right), \S \mathrm{I}$, is fulfilled for $y(x)$ and $Y(x)=y(x-h)$ at $x=q$. This means that

$$
\lim _{x \rightarrow q+0}\left\{y^{\prime}(x) y(x-h)-y(x) y^{\prime}(x-h)\right\}=0, h=q-x_{0},
$$

or, since the first term tends to zero and $y(x) /(x-q)$ tends to a limit different from zero,

$$
\lim _{x \rightarrow q+0}(x-q) y^{\prime}(x-h)=\lim _{x \rightarrow x_{0}+0}\left(x-x_{0}\right) y^{\prime}(x)=0 .
$$

3. In what follows we apply these theorems to the Legendre differential equation in the form

* We suppose, of course, the existence of at least three zeros in the interval considered. 
(A)

$$
z^{\prime \prime}+\left\{\left(n+\frac{1}{2}\right)^{2}+(2 \sin \theta)^{-2}\right\} z=0, \quad z=(\sin \theta)^{1 / 2} P_{n}(\cos \theta),
$$

to the Bessel differential equation

$$
u^{\prime \prime}+\left\{1+(2 \theta)^{-2}\right\} u=0, \quad u=\theta^{1 / 2} J_{0}(\theta),
$$

to the differential equation of the ultraspherical polynomials

$$
\text { (C) } z_{\mu}^{\prime \prime}+\left\{(n+\mu)^{2}+\mu(1-\mu)(\sin \theta)^{-2}\right\} z_{\mu}=0, \quad z_{\mu}=(\sin \theta)^{\mu} P_{n}^{(\mu)}(\cos \theta) \text {, }
$$

and to the general Bessel differential equation

$$
u_{\lambda}^{\prime \prime}+\left\{1+\left(1-4 \lambda^{2}\right)(2 \theta)^{-2}\right\} u_{\lambda}=0, \quad u_{\lambda}=\theta^{1 / 2} J_{\lambda}(\theta) .
$$

The equations (A) and (C) are satisfied in the open interval $0, \pi ;(B)$ and $(\mathrm{D})$ are valid for $\theta>0$.

\section{LEgENDRE POLYNOMIALS}

1. We compare (A), $z=Y$, with the solution $y=\sin \left(n+\frac{1}{2}\right)\left(x-x_{0}\right)$ of

$$
y^{\prime \prime}+\left(n+\frac{1}{2}\right)^{2} y=0 .
$$

This gives at once the existence of at least one zero of $(\sin \theta)^{1 / 2} P_{n}(\cos \theta)$ in every interval of length $\pi /\left(n+\frac{1}{2}\right)$, especially in the intervals

$$
\frac{\nu-1}{n+\frac{1}{2}} \pi<\theta<\frac{\nu}{n+\frac{1}{2}} \pi \quad(\nu=1,2, \cdots, n)
$$

[in the first interval condition $\left(3^{\prime}\right), \S \mathrm{I}$, is satisfied]. Consequently every interval contains exactly one zero $\theta_{v}$, and

$$
\frac{\nu-1}{n+\frac{1}{2}} \pi<\theta_{\nu}<\frac{\nu}{n+\frac{1}{2}} \pi \quad(\nu=1,2, \cdots, n) .
$$

The lower estimate can be improved by means of the symmetric property

$$
\theta_{\nu}=\pi-\theta_{n+1-\nu}>\pi-\frac{n+1-\nu}{n+\frac{1}{2}} \pi=\frac{\nu-\frac{1}{2}}{n+\frac{1}{2}} \pi,
$$

so that we obtain the inequalities of Bruns.

2. We now prove first the upper estimate (3) of the Introduction. Since $(2 \sin \theta)^{-2}$ decreases in $0<\theta \leqq \pi / 2$, Sturm's theorem $(\$ I, 2)$ asserts the convexity of the sequence of $\theta_{v}$ [(5) of the Introduction]. For even values of $n$ the first remark in $\$ I, 2$ [cf. (6)] must be used in the interval $\theta_{n / 2}<\theta<\theta_{n / 2+1}$. Hence the convexity of the sequence

$$
\theta_{\nu}^{\prime}=\theta_{\nu}-\frac{\nu}{n+1} \pi \quad(\nu=0,1,2, \cdots,[n / 2]+1)
$$


also follows. Now a convex sequence attains its maximum only at the end points of any interval, that is to say, always for the first or the last value of $\nu$. Since $\theta_{0}^{\prime}=0$ and, for $n$ odd, $\theta_{(n+1) / 2}^{\prime}=0$, this gives at once the upper bound for $n$ odd. Let further $n$ be even; then $\theta_{n / 2}^{\prime}+\theta_{n / 2+1}^{\prime}=0$, so that there are only the possibilities

$$
\theta_{n / 2}^{\prime}<0, \theta_{n / 2+1}^{\prime}>0 ; \theta_{n / 2}^{\prime}>0, \theta_{n / 2+1}^{\prime}<0 ; \theta_{n / 2}^{\prime}=\theta_{n / 2+1}^{\prime}=0 .
$$

Each of the last two cases is impossible: $\theta_{0}^{\prime}=0, \theta_{n / 2+1}^{\prime}<0$ implies $\theta_{n / 2}^{\prime}<0$ and the third assumption would give, since $\theta_{a}^{\prime}=0$, that $\theta_{\nu}^{\prime}=0$ for each $\nu$, i.e., the identity of $P_{n}(\cos \theta)$ and $\sin (n+1) \theta / \sin \theta$. But $\theta_{n / 2}^{\prime}<0$ yields $\theta_{\nu}^{\prime}<0, \nu=1$, $2, \cdots, n / 2$, therefore we have again the upper bound obtained above.

We base the proof of the lower bound on the inequality

$$
\theta_{\nu}-\theta_{\nu-1}<\pi /\left(n+\frac{1}{2}\right) \quad\left(\nu=1,2, \cdots, n ; \theta_{0}=0\right)
$$

which is a consequence of the comparison of (A) with (1). We put now

$$
\theta_{\nu}^{\prime \prime}=\theta_{\nu}-\frac{\nu-\frac{1}{4}}{n+\frac{1}{2}} \pi
$$

according to (4) we have $\theta_{\nu}^{\prime \prime}-\theta_{-1}^{\prime \prime}<0$, so that $\theta_{\nu}^{\prime \prime}$ is decreasing. For $n$ odd, $\theta_{(n+1) / 2}^{\prime \prime}=0$, consequently $\theta_{\nu}^{\prime \prime}>0, \nu<(n+1) / 2$. For $n$ even it is sufficient to prove $\theta_{n / 2}^{\prime \prime}>0$. This follows from (4), because

$$
\theta_{n / 2+1}-\theta_{n / 2}=\pi-\theta_{n / 2}-\theta_{n / 2}<\pi /\left(n+\frac{1}{2}\right) \text {. }
$$

Thus our theorem is completely proved.

III. Bessel function $J_{0}(\theta)$ AND LEgendRe polynomials Again

1. We compare (B) first with $y^{\prime \prime}+y=0$. This gives at once the existence of an infinite number of zeros of $\theta^{1 / 2} J_{0}(\theta)$ :

$$
0=j_{0}<j_{1}<j_{2}<\cdots,
$$

for which

$$
j_{\nu}-j_{\nu-1}<\pi \text { and } j_{\nu}<\nu \pi \quad(\nu=1,2,3, \cdots) .
$$

Moreover the differences $j_{v}-j_{v-1}$ are monotonically increasing, so that the sequence $\left\{j_{\nu}\right\}$ is convex. These facts are well known.*

Using (2) we see that $j_{\nu}-\nu \pi$ is decreasing, therefore

$$
j_{\nu}-\nu \pi \leqq j_{1}-\pi=0.764 \cdots \pi-\pi,
$$

whence

${ }^{*}$ Cf. Sturm 9, pp. 174-175. 


$$
j_{\nu} \leqq\left(\nu-\frac{1}{4}\right) \pi+0.014 \cdots \pi .
$$

2. Now we compare (A), $z=Y$, with

$$
y^{\prime \prime}+\left\{\left(n+\frac{1}{2}\right)^{2}+(2 \theta)^{-2}\right\} y=0,
$$

which has the solution $y=\theta^{1 / 2} J_{0}\left[\left(n+\frac{1}{2}\right) \theta\right], 0<\theta<\pi$ [condition $\left(3^{\prime}\right), \S \mathrm{I}$, at $\theta=a=0$ is satisfied]. Since $\sin \theta<\theta$ we obtain the existence of at least one zero of $P_{n}(\cos \theta)$ in every interval $j_{\nu-1} /\left(n+\frac{1}{2}\right), j_{\nu} /\left(n+\frac{1}{2}\right), \nu=1,2, \cdots, n$. The fact that $j_{n} /\left(n+\frac{1}{2}\right)<\pi$, implies the existence of exactly one zero in each of these intervals, so that

$$
j_{\nu-1} /\left(n+\frac{1}{2}\right)<\theta_{\nu}<j_{\nu} /\left(n+\frac{1}{2}\right) \quad(\nu=1,2, \cdots, n) .
$$

The upper estimate is particularly important. It is better than the upper estimate of Bruns. It yields at once a lower bound for $j_{\nu}$. In fact, let $\nu$ be any positive integer, and $n=2 \nu-1$. Then $\nu=(n+1) / 2, \theta_{\nu}=\pi / 2$. We have therefore $\pi / 2<j_{\nu} /\left(2 \nu-\frac{1}{2}\right)$, that is, in view of $(3), *$

$$
\left(\nu-\frac{1}{4}\right) \pi<j_{\nu} \leqq\left(\nu-\frac{1}{4}\right) \pi+0.014 \cdots \pi \quad(\nu=1,2,3, \cdots) .
$$

The upper estimate (4) for $\theta_{\nu}=\theta_{\nu}(n)$ is the best possible for fixed $\nu, n \rightarrow \infty$. Indeed it is known that

$$
\lim _{n \rightarrow \infty}\left(n+\frac{1}{2}\right) \theta_{\nu}=j_{\nu}
$$

3. The first inequality in (4) is not particularly sharp. To obtain a better one, we use the elementary inequality $\dagger$

$$
(\sin \theta)^{-2}-\theta^{-2} \leqq 1-(2 / \pi)^{2}=k, \quad 0<\theta \leqq \pi / 2,
$$

and compare (A), $z=y$, with

$$
Y^{\prime \prime}+\left\{\left(n+\frac{1}{2}\right)^{2}+k / 4+(2 \theta)^{-2}\right\} Y=0
$$

instead of $\left(\mathrm{B}^{\prime}\right)$. Thus we obtain

$$
\theta_{\nu}>j_{\nu} /\left[\left(n+\frac{1}{2}\right)^{2}+k / 4\right]^{1 / 2}, 0<\theta_{\nu} \leqq \pi / 2, k / 4=0.148678816 \cdots .
$$

The same argument gives a sharper inequality for the zeros in the interval $0<\theta \leqq v$, where $v$ is a fixed positive number, $v<\pi / 2$; we then obtain (7) with a constant $k_{v}$ instead of $k$, where $k_{v}=(\sin v)^{-2}-v^{-2}$. For example,

$$
\begin{aligned}
& \frac{1}{4} k_{\pi / 4}=0.094715264 \cdots, \\
& \frac{1}{4} k_{\pi / 6}=0.088109344 \cdots .
\end{aligned}
$$

* Cf. Watson 11, p. 489-490; there it is shown that the positive zeros of $J_{0}(\theta)$ lie in the intervals $\left(\nu-\frac{1}{4}\right) \pi,\left(\nu-\frac{1}{8}\right) \pi$ (theorem of Schafheitlin).

$\dagger$ The function on the left side is increasing in $0, \pi / 2$. 
Formula (7) gives also an upper bound for $j_{\nu}$, putting as before $n=2 \nu-1$, $\nu=(n+1) / 2, \theta_{\nu}=\pi / 2$ :

$$
\begin{aligned}
j_{\nu} & <\frac{\pi}{2}\left[\left(2 \nu-\frac{1}{2}\right)^{2}+\frac{k}{4}\right]^{1 / 2}=\left(\nu-\frac{1}{4}\right) \pi\left[1+\frac{k / 4}{\left(2 \nu-\frac{1}{2}\right)^{2}}\right]^{1 / 2 *} \\
& <\left(\nu-\frac{1}{4}\right) \pi+\frac{k \pi}{32\left(\nu-\frac{1}{4}\right)} .
\end{aligned}
$$

This inequality for $\nu=1$ is not as good as (3); it is much better, however, for $\nu \geqq 2$ and especially for large $\nu$.

IV. Ultraspherical Polynomials AND geNERAL BESSEL FunCtions

1. We consider equation (C) in the "principal case" $0<\mu<1$. The same argument as in the special case $\mu=\frac{1}{2}$ (§II, 1) gives, with the same notation as was used in that case,

$$
\frac{\nu-1}{n+\mu} \pi<\theta_{\nu}<\frac{\nu}{n+\mu} \pi \quad(\nu=1,2, \cdots, n),
$$

from which we obtain by means of the symmetric property

$$
\frac{\nu-(1-\mu)}{n+\mu} \pi<\theta_{\nu}<\frac{\nu}{n+\mu} \pi \quad(\nu=1,2, \cdots, n) .
$$

These are the inequalities corresponding to those of Bruns.

2. We have further

$$
\frac{\nu-\frac{1}{2}(1-\mu)}{n+\mu} \pi<\theta_{\nu}<\frac{\nu}{n+1} \pi \quad(\nu=1,2, \cdots, n),
$$

corresponding to the Markoff-Stieltjes inequalities. The proof is the same as in $\S I I, 2$; it is based on the convexity of the sequences $\theta_{\nu}$, and

$$
\theta_{\nu}-\frac{\nu-\frac{1}{2}(1-\mu)}{n+\mu} \pi \quad(\nu=0,1,2, \cdots,[n / 2]+1),
$$

and on the inequality

$$
\theta_{\nu}-\theta_{\nu-1}<\pi /(n+\mu) \quad(\nu=1,2, \cdots, n) .
$$

3. In the "principal case" $-\frac{1}{2}<\lambda<\frac{1}{2}$ we obtain similar results for the zeros $j_{\nu}(\lambda)$ of $\theta^{1 / 2} J_{\lambda}(\theta)$ as in $\S I I I, 1$. We have in particular

$$
j_{\nu}(\lambda)-j_{\nu-1}(\lambda)<\pi, j_{\nu}(\lambda)<\nu \pi \quad\left(\nu=1,2,3, \cdots, j_{0}(\lambda)=0\right),
$$

and

$$
{ }^{*}(1+x)^{1 / 2}<1+x / 2, x>0 \text {. }
$$




$$
j_{\nu}(\lambda) \leqq(\nu-1) \pi+j_{1}(\lambda) .
$$

We further compare (C) with (D) putting $\mu=\lambda+\frac{1}{2}$; we obtain [condition $\left(3^{\prime}\right), \S I$, is satisfied]

$$
j_{\nu-1}(\lambda) /(n+\mu)<\theta_{\nu}<j_{\nu}(\lambda) /(n+\mu) \quad(\nu=1,2, \cdots, n) .
$$

The upper estimate here is the best possible in a sense analogous to $\$ I I I,(6)$. We obtain from it as before $\pi / 2<j_{\nu}(\lambda) /(2 \nu-1+\mu)$, so that*

$$
\left(\nu+\lambda / 2-\frac{1}{4}\right) \pi<j_{\nu}(\lambda)<(\nu-1) \pi+j_{1}(\lambda)(<\nu \pi) \quad(\nu=1,2,3, \cdots) .
$$

Remarks. Comparison of $(\sin \theta)^{\mu} P_{n}^{(\mu)}(\cos \theta)$ with $\theta^{1 / 2} J_{-\lambda}(\theta)$ [for $\lambda=0$ with the Bessel function of the second kind $\left.Y_{0}(\theta)\right]$ is also possible. Condition (3), $\S \mathrm{I}$, is now satisfied only if $\lambda<0$. We consequently obtain

$$
\theta_{\nu}<j_{\nu}(-\lambda) /(n+\mu) \text { if } \lambda<0 ; \theta_{\nu}<j_{\nu+1}(-\lambda) /(n+\mu) \text { if } \lambda \geqq 0 \text {. }
$$

On the other hand the ordinary form of Sturm's theorem gives at once $j_{\nu}(-\lambda)>j_{\nu}(\lambda)$ in the first case, $j_{\nu+1}(-\lambda)>j_{\nu}(\lambda)$ in the second, so that these bounds are less precise than (8).

The lower estimate in (8), on the other hand, is always valid with $-\lambda$ in place of $\lambda$. For negative $\lambda$ this result is better than (8).

4. We obtain a better lower bound of $\theta_{\nu}$ than that given in (8) in a way similar to that of $\$ I I I, 3$ :

$$
\theta_{\nu}>j_{\nu}(\lambda) /\left[(n+\mu)^{2}+k \mu(1-\mu)\right]^{1 / 2}, \quad 0<\theta_{\nu} \leqq \pi / 2,
$$

where $k$ has the same meaning as in $\$ I I I, 3$. Hence it follows as before that

$$
i_{\nu}(\lambda)<\left(\nu+\lambda / 2-\frac{1}{4}\right) \pi+(k / 8)\left(\frac{1}{4}-\lambda^{2}\right) \frac{\pi}{\nu+\lambda / 2-\frac{1}{4}} .
$$

For large values of $\nu$, this bound is better than either the upper bound in (9) or that due to Schafheitlin, quoted in the last footnote.

\section{Part 2. TRIgonometric POLYNomials}

\section{Distribution OF zeros}

1. Let $\lambda_{0}, \lambda_{1}, \lambda_{2}, \cdots, \lambda_{m}$ be non-negative numbers satisfying the inequalities

* Cf. Watson 11, pp. 490-491, where a theorem of Schafheitlin (in extended form) is stated: the zeros of $J_{\lambda}(\theta)$ lie in the intervals

$$
\left(\nu+\lambda / 2-\frac{1}{4}\right) \pi \leqq \theta \leqq\left(\nu+\lambda / 4-\frac{1}{8}\right) \pi \quad\left(\nu=1,2,3, \cdots,-\frac{1}{2}<\lambda<\frac{1}{2}\right) .
$$

The upper bound (9) is better than this, since

$$
j_{1}(\lambda)<\left(\lambda / 4+\frac{7}{8}\right) \pi, \quad-\frac{1}{2}<\lambda<\frac{1}{2},
$$

for $J_{\lambda}\left[\left(\lambda / 4+\frac{7}{8}\right) \pi\right]<0$ (cf. Watson 11, p. 491, $\phi=\pi / 8$ ). 


$$
\lambda_{0}>\lambda_{1} \geqq \lambda_{2} \geqq \cdots \geqq \lambda_{m} \geqq 0 .
$$

We shall prove that the trigonometric expressions

$$
\begin{aligned}
& p(t)=\lambda_{0} \cos m t+\lambda_{1} \cos (m-1) t+\cdots+\lambda_{m-1} \cos t+\lambda_{m}, \\
& q(t)=\lambda_{0} \sin m t+\lambda_{1} \sin (m-1) t+\cdots+\lambda_{m-1} \sin t, \\
& r(t)=\lambda_{0} \cos \left(m+\frac{1}{2}\right) t+\lambda_{1} \cos \left(m-\frac{1}{2}\right) t+\cdots+\lambda_{m-1} \cos \frac{3}{2} t+\lambda_{m} \cos \frac{1}{2} t, \\
& s(t)=\lambda_{0} \sin \left(m+\frac{1}{2}\right) t+\lambda_{1} \sin \left(m-\frac{1}{2}\right) t+\cdots+\lambda_{m-1} \sin \frac{3}{2} t+\lambda_{m} \sin \frac{1}{2} t
\end{aligned}
$$

have only real and simple zeros which are regularly distributed in the following sense. Denoting by $t_{1}, t_{2}, t_{3}, \cdots$ the zeros in question in the interval $0<t<\pi$, in increasing order, we have for $p(t), q(t), r(t), s(t)$ respectively

$$
\begin{array}{lc}
\frac{\mu-\frac{1}{2}}{m+\frac{1}{2}} \pi<t_{\mu}<\frac{\mu+\frac{1}{2}}{m+\frac{1}{2}} \pi & (\mu=1,2, \cdots, m), \\
\frac{\mu}{m+\frac{1}{2}} \pi<t_{\mu}<\frac{\mu+1}{m+\frac{1}{2}} \pi \quad(\mu=1,2, \cdots, m-1), \\
\frac{\mu-\frac{1}{2}}{m+1} \pi<t_{\mu}<\frac{\mu+\frac{1}{2}}{m+1} \pi \quad(\mu=1,2, \cdots, m), \\
\frac{\mu}{m+1} \pi<t_{\mu}<\frac{\mu+1}{m+1} \pi \quad(\mu=1,2, \cdots, m) .
\end{array}
$$

Besides these zeros in the interval $0<t<\pi$, there are, of course, the other zeros $\pm t_{\mu}+2 h \pi$; moreover $q(t)$ has the zeros $h \pi, r(t)$ the zeros $(2 h+1) \pi, s(t)$ the zeros $2 h \pi$. Here $h$ is an arbitrary integer. All these zeros are simple.

As another formulation, we have in the open intervals

$$
\begin{array}{ll}
\left(\frac{\mu-\frac{1}{2}}{m+\frac{1}{2}} \pi, \frac{\mu+\frac{1}{2}}{m+\frac{1}{2}} \pi\right), & \left(\frac{\mu}{m+\frac{1}{2}} \pi, \frac{\mu+1}{m+\frac{1}{2}} \pi\right), \\
\left(\frac{\mu-\frac{1}{2}}{m+1} \pi, \frac{\mu+\frac{1}{2}}{m+1} \pi\right), & \left(\frac{\mu}{m+1} \pi, \frac{\mu+1}{m+1} \pi\right),
\end{array}
$$

respectively, exactly one zero of $p(t), q(t), r(t), s(t) ; \mu$ runs here over all integer values except

$$
\begin{array}{ll}
\mu \equiv 0(\bmod 2 m+1), & \mu \equiv-1,0(\bmod 2 m+1), \\
\mu \equiv 0(\bmod 2 m+2), & \mu \equiv-1,0(\bmod 2 m+2)
\end{array}
$$

in the corresponding cases. In the second and fourth case we further have the trivial zeros $t=0, \pm 2 \pi, \pm 4 \pi, \cdots$; all zeros (4) are "non-trivial" except in the intervals $\mu \equiv m(\bmod 2 m+1)$ in the second, $\mu \equiv m+1(\bmod 2 m+2)$ in the third case, containing the zeros $t= \pm \pi, \pm 3 \pi, \cdots$. 
2. The proof of the inequalities just formulated is very simple and can be based entirely on the classical fact that the trigonometric sums

$$
\sigma_{m}(t)=\sin \frac{1}{2} t+\sin \frac{3}{2} t+\cdots+\sin \left(m+\frac{1}{2}\right) t \quad(m=0,1,2,3, \cdots)
$$

are all non-negative for $0<t<2 \pi$. This property is well known from Fejér's summability theory of Fourier series, in which it plays a decisive role.

We have

$$
\begin{aligned}
-\Im e^{-i(m+1 / 2) t}\{p(t)+i q(t)\} & =-\Im e^{-i(m+1) t}\{r(t)+i s(t)\} \\
& =\sum_{k=0}^{m} \lambda_{k} \sin \left(k+\frac{1}{2}\right) t .
\end{aligned}
$$

Partial summation shows at once that the last expression is non-negative for $0<t<2 \pi$. More precisely, it remains there decidedly positive because

$$
\begin{aligned}
\sum_{k=0}^{m} \lambda_{k} \sin \left(k+\frac{1}{2}\right) t= & \left(\lambda_{0}-\lambda_{1}\right) \sigma_{0}(t)+\left(\lambda_{1}-\lambda_{2}\right) \sigma_{1}(t)+\cdots \\
& +\left(\lambda_{m-1}-\lambda_{m}\right) \sigma_{m-1}(t)+\lambda_{m} \sigma_{m}(t),
\end{aligned}
$$

the first term being positive. From this remark we deduce the important inequalities

$$
\begin{aligned}
& p(t) \sin \left(m+\frac{1}{2}\right) t-q(t) \cos \left(m+\frac{1}{2}\right) t>0, \\
& r(t) \sin (m+1) t-s(t) \cos (m+1) t>0, \quad 0<t<2 \pi,
\end{aligned}
$$

so that for the values of $\mu$ mentioned in (3);

$$
\begin{aligned}
\operatorname{sgn} p\left(\frac{\mu-\frac{1}{2}}{m+\frac{1}{2}} \pi\right) & =\operatorname{sgn} q\left(\frac{\mu}{m+\frac{1}{2}} \pi\right)=\operatorname{sgn} r\left(\frac{\mu-\frac{1}{2}}{m+1} \pi\right) \\
& =\operatorname{sgn} s\left(\frac{\mu}{m+1} \pi\right)=(-1)^{\mu+1}
\end{aligned}
$$

and this gives our assertion.

3. Under more restrictive conditions than (1), sharper inequalities can be stated. Let the coefficients satisfy, for example, the following conditions:

$$
2 \lambda_{0}-\lambda_{1}>\lambda_{1}-\lambda_{2} \geqq \lambda_{2}-\lambda_{3} \geqq \cdots \geqq \lambda_{m-1}-\lambda_{m} \geqq \lambda_{m} \geqq 0 .
$$

(This is always satisfied if the sequence $\lambda_{0}, \lambda_{1}, \cdots, \lambda_{m}, 0,0$ is convex and not identically zero.) We then prove that

$$
\frac{\mu}{m} \pi, \frac{\mu+\frac{1}{2}}{m} \pi, \frac{\mu}{m+\frac{1}{2}} \pi, \frac{\mu+\frac{1}{2}}{m+\frac{1}{2}} \pi
$$


can be taken as upper bounds of $t_{\mu}$, instead of those in (3) respectively. To show this, it is sufficient to prove that

$$
\begin{aligned}
\operatorname{sgn} p\left(\frac{\mu}{m} \pi\right) & =\operatorname{sgn} q\left(\frac{\mu+\frac{1}{2}}{m} \pi\right)=\operatorname{sgn} r\left(\frac{\mu}{m+\frac{1}{2}} \pi\right) \\
& =\operatorname{sgn} s\left(\frac{\mu+\frac{1}{2}}{m+\frac{1}{2}} \pi\right)=(-1)^{\mu} .
\end{aligned}
$$

We have

$$
\left(1-e^{i t}\right) \sum_{k=0}^{m} \lambda_{k} e^{i(m-k) t}=-\lambda_{0} e^{i(m+1) t}+\sum_{k=0}^{m-1}\left(\lambda_{k}-\lambda_{k+1}\right) e^{i(m-k) t}+\lambda_{m} .
$$

so that

$$
\begin{aligned}
- & \Im e^{-i(m+1 / 2) t}\left(1-e^{i t}\right) \sum_{k=0}^{m} \lambda_{k} e^{i(m-k) t} \\
= & \lambda_{0} \sin \frac{1}{2} t+\sum_{k=0}^{m-1}\left(\lambda_{k}-\lambda_{k+1}\right) \sin \left(k+\frac{1}{2}\right) t+\lambda_{m} \sin \left(m+\frac{1}{2}\right) t \\
= & \left(2 \lambda_{0}-\lambda_{1}\right) \sin \frac{1}{2} t+\left(\lambda_{1}-\lambda_{2}\right) \sin \frac{3}{2} t+\cdots \\
& +\left(\lambda_{m-1}-\lambda_{m}\right) \sin \left(m-\frac{1}{2}\right) t+\lambda_{m} \sin \left(m+\frac{1}{2}\right) t .
\end{aligned}
$$

The positivity of this trigonometric polynomial in $0, \pi$ (even in $0,2 \pi$ ) is a consequence of our condition, so that

$$
p(t) \cos m t+q(t) \sin m t>0, \quad 0<t<2 \pi .
$$

Similarly it is shown that

$$
r(t) \cos \left(m+\frac{1}{2}\right) t+s(t) \sin \left(m+\frac{1}{2}\right) t>0, \quad 0<t<2 \pi .
$$

Thus our theorem is established.

4. The reality of the zeros of

$$
\alpha p(t)+\beta q(t), \quad \alpha r(t)+\beta s(t)
$$

follows in the same way as in 2, provided that the inequalities (1) are satisfied; here $\alpha, \beta$ are arbitrary real constants not vanishing simultaneously. For these zeros inequalities similar to those given above hold; they are all simple.

To prove this statement, put $\alpha+i \beta=\rho e^{i \delta}(\rho>0, \delta$ real). We obtain from (9) for $\left(m+\frac{1}{2}\right) t=\left(\mu-\frac{1}{2}\right) \pi+\delta, 0<t<2 \pi$,

$$
(-1)^{\mu+1}\{p(t) \cos \delta+q(t) \sin \delta\}>0,
$$

so that

$$
\{\operatorname{sgn} \alpha p(t)+\beta q(t)\}=(-1)^{\mu+1} .
$$


We can assume $0<\delta<\pi, \delta \neq \pi / 2$. The last result gives immediately the existence of at least one (consequently of exactly one) zero in each interval

$$
\begin{aligned}
& \frac{\left(\mu-\frac{1}{2}\right) \pi+\delta}{m+\frac{1}{2}}<t<\frac{\left(\mu+\frac{1}{2}\right) \pi+\delta}{m+\frac{1}{2}}, \mu=1,2, \cdots, 2 m, \text { if } 0<\delta<\pi / 2, \\
& \frac{\left(\mu-\frac{3}{2}\right) \pi+\delta}{m+\frac{1}{2}}<t<\frac{\left(\mu-\frac{1}{2}\right) \pi+\delta}{m+\frac{1}{2}}, \mu=1,2, \cdots, 2 m, \text { if } \pi / 2<\delta<\pi .
\end{aligned}
$$

These are the zeros in the interval $0<t<2 \pi$. All zeros are real and simple, lying in the intervals (19), where $\mu$ runs over all integer values, $\mu \neq 0(\bmod$ $2 m+1)$.

Trigonometric polynomials $\alpha r(t)+\beta s(t)$ can be treated in an analogous way with a similar result.

\section{ON a theorem of Pólya}

The elementary inequalities of the preceding paragraph lead in a direct way to a theorem of Pólya [7], giving even a slightly more precise result. We consider the entire functions of $z$

$$
U(z)=\int_{0}^{1} f(x) \cos z x d x, \quad V(z)=\int_{0}^{1} f(x) \sin z x d x
$$

and we prove the following theorem:

Let $f(x)$ be non-negative, monotonically non-decreasing and not identically zero in $0<x<1$; further, let the integral $\int^{1} f(x) d x$ exist. Let $\alpha$ and $\beta$ denote real constants not both zero, $\alpha+i \beta=\rho e^{i \delta}, \rho>0,0<\delta \leqq \pi$. The entire function $\alpha U(z)+\beta V(z)$ has only real and simple zeros; every interval

$$
\left(\mu-\frac{1}{2}\right) \pi+\delta, \quad\left(\mu+\frac{1}{2}\right) \pi+\delta \quad(\mu=0, \pm 1, \pm 2, \cdots),
$$

except that with $z=0,{ }^{*}$ contains exactly one zero as inner point.

The only exception is the case in which $f(x)$ is a step function with jumps at the points of the form $1-2 \pi h /\left[\left(\mu-\frac{1}{2}\right) \pi+\delta\right], h$ and $\mu$ integers. In this case the zeros are also real and lie in the closed intervals (2).

The proof is based (in a somewhat different form from that in the paper of Pólya 7, p. 361) on the trigonometric expressions

$$
\begin{aligned}
& U_{m}(z)=\frac{1}{m} \sum_{k=0}^{m} f\left(\frac{k}{m+\frac{1}{2}}\right) \cos \frac{k}{m+\frac{1}{2}} z, \\
& V_{m}(z)=\frac{1}{m} \sum_{k=0}^{m} f\left(\frac{k}{m+\frac{1}{2}}\right) \sin \frac{k}{m+\frac{1}{2}} z,
\end{aligned}
$$

${ }^{*}$ For $\alpha=\cos \delta=0$, i.e., for $V(z)$ itself, we have two exceptional intervals, namely $-\pi, 0$ and $0, \pi$ with the single simple zero $z=0$. 
the symbol $\sum^{\prime}$ meaning that the highest term $k=m$ is to be multiplied by $1+m^{-1}$ or more generally by a factor $>1$ tending to 1 for $m \rightarrow \infty$. These expressions tend respectively to $U(z)$ and $V(z)$ uniformly in an arbitrary finite region of the z-plane. The same is valid for $\alpha U_{m}(z)+\beta V_{m}(z)$. Hence we obtain, by means of the results of $\S \mathrm{V}, 4$, and of a well known theorem of Hurwitz (used also by Pólya), that all the zeros of $\alpha U(z)+\beta V(z)$ lie in the closed intervals (2).

Zeros in the inner part of these intervals are of course always simple. The only double zeros must have the form $z=z_{0}=\left(\mu_{0}-\frac{1}{2}\right) \pi+\delta$ ( $\mu_{0}$ integer). Now we have*

$$
\begin{aligned}
& -\Im e^{-i z}\{U(z)+i V(z)\} \\
& =-\Im e^{-i z} \int_{0}^{1} f(x) e^{i z x} d x \\
& =\int_{0}^{1} f(1-x) \sin z x d x=\int_{0}^{\infty} g(x) \sin z x d x,
\end{aligned}
$$

putting $g(x)=f(1-x)$ for $0<x<1, g(x)=0$ for $x>1$. The last integral can be written for $z>0$ in the form (cf. Pólya 7, p. 378)

$$
\int_{0}^{\pi / z} \sin z x\{g(x)-g(x+\pi / z)+g(x+2 \pi / z)-g(x+3 \pi / z)+\cdots\} d x,
$$

which is positive "in general" for all values of $z, z>0$. Consequently we obtain for $z=z_{0}=\left(\mu_{0}-\frac{1}{2}\right) \pi+\delta$

$$
(-1)^{\mu_{0}+1}\left\{U\left(z_{0}\right) \cos \delta+V\left(z_{0}\right) \sin \delta\right\}>0,
$$

so that $z_{0}$ is not a zero for $U(z) \cos \delta+V(z) \sin \delta$. Incidentally, this argument yields at once the existence of at least one zero in the intervals (2).

Zeros of the form $z=z_{0}=\left(\mu_{0}-\frac{1}{2}\right) \pi+\delta, z_{0}>0, \uparrow$ can occur only if the integral

(5) vanishes for $z=z_{0}$, that is, if

$$
\begin{aligned}
g(x)-g\left(x+\pi / z_{0}\right)=0, g\left(x+2 \pi / z_{0}\right)-g\left(x+3 \pi / z_{0}\right) & =0, \cdots, \\
& 0<x<\pi / z_{0} .
\end{aligned}
$$

This means that $g(x)=f(1-x)$ is a step function with jumps at the points $2 \pi / z_{0}, 4 \pi / z_{0}, 6 \pi / z_{0}, \cdots$.

Indeed under this assumption we have

* The following argument corresponds to the treatment of the "algebraic" case given in $\S \mathrm{V}, 2$.

$\dagger$ We can assume $z_{0}>0$ because (4) is an odd function. 
$U(z) \cos \delta+V(z) \sin \delta=\int_{0}^{1} f(x) \cos (z x-\delta) d x=\int_{0}^{1} g(x) \cos (z x-z+\delta) d x$

$$
\begin{gathered}
=\sum_{k=0}^{m-1} g_{k} \int_{k d}^{(k+1) d} \cos (z x-z+\delta) d x, \\
g_{0} \geqq g_{1} \geqq \cdots \geqq g_{m-1} \geqq 0, g_{0}>0 ; d=2 \pi / z_{0}, m=\left[z_{0} /(2 \pi)\right] .
\end{gathered}
$$

Now the last sum is

$$
\begin{aligned}
& =\sum_{k=0}^{m-1} g_{k} \frac{\sin (z(k+1) d-z+\delta)-\sin (z k d-z+\delta)}{z} \\
& =\frac{2 \sin (z d / 2)}{z} \sum_{k=0}^{m-1} g_{k} \cos \left(z\left(k+\frac{1}{2}\right) d-z+\delta\right) .
\end{aligned}
$$

Both factors have here the simple zero $z=z_{0}$.

The theorem proved in $\S \mathrm{V}, 3$, gives similarly the results of Pólya for convex and increasing $f(x)$.

VII. TRIgonometric POLYNOMIALS OF THE LEgENDRE TYPE

1. Let

$$
\alpha_{0}, \alpha_{1}, \alpha_{2}, \cdots, \alpha_{n}, \cdots
$$

be a given sequence of positive numbers. The cosine polynomials

$$
\begin{aligned}
f_{n}(\theta) & =\alpha_{0} \alpha_{n} \cos n \theta+\alpha_{1} \alpha_{n-1} \cos (n-2) \theta+\cdots+\left\{\begin{array}{c}
\frac{1}{2} \alpha_{n / 2}^{2}, \text { if } n \text { is even, } \\
\alpha_{(n-1) / 2} \alpha_{(n+1) / 2} \cos \theta, \\
\text { if } n \text { is odd }
\end{array}=\sum_{k=0}^{[n / 2]} \alpha_{k} \alpha_{n-k} \cos (n-2 k) \theta^{*}\right.
\end{aligned}
$$

have been considered by Fejér [3]; Legendre polynomials $P_{n}(\cos \theta)$ are particular cases for

$$
\alpha_{n}=g_{n}=\frac{1 \cdot 3 \cdots(2 n-1)}{2 \cdot 4 \cdots 2 n}=(1 / \pi) \int_{0}^{1} x^{n-1 / 2}(1-x)^{-1 / 2} d x .
$$

In what follows we use also the corresponding sine polynomials

$$
g_{n}(\theta)=\sum_{k=0}^{[n / 2]} \alpha_{k} \alpha_{n-k} \sin (n-2 k) \theta .
$$

I have proved in a previous paper [10] that all zeros of $f_{n}(\theta)$ are real and simple provided that the sequence

$$
\alpha_{1} / \alpha_{0}, \alpha_{2} / \alpha_{1}, \alpha_{3} / \alpha_{2}, \cdots, \alpha_{n} / \alpha_{n-1}, \cdots
$$

\footnotetext{
* For $n$ even, the last term $\nu=n / 2$ is to be multiplied by $\frac{1}{2}$.
} 
is monotonically increasing. Under this condition the coefficients of (2) are monotonically increasing. On putting $2 \theta=t$ and $m=n / 2$ or $m=(n-1) / 2$, $f_{n}(\theta)$ becomes of the type $p(t)$ or $r(t)$ in $\S \mathrm{V},(2)$, respectively. The inequalities of $\S \mathrm{V},(3)$, give at once the following information about the zeros $\theta_{\nu}$ of $f_{n}(\theta)$, $0<\theta_{1}<\theta_{2}<\cdots<\theta_{n}<\pi$ :

$$
\frac{\nu-\frac{1}{2}}{n+1} \pi<\theta_{\nu}<\frac{\nu+\frac{1}{2}}{n+1} \pi \quad(\nu=1,2, \cdots, n) .
$$

2. The inequalities just obtained are not so precise as those of Bruns. By making some restrictions on the sequence $\left\{\alpha_{n}\right\}$, they can be improved. We show the possibility of deriving by this very elementary method the lower estimate not only in the theorem of Bruns but also in that of MarkoffStieltjes.

Let the sequence $\left\{\alpha_{n}\right\}$ be of the form

$$
\alpha_{n}=\int_{0}^{1} x^{n} f(x) d x
$$

where $f(x)$ is non-negative and integrable in the Lebesgue sense with $\alpha_{0}>0$.

The sequence $\alpha_{n}=g_{n}$, corresponding to the Legendre polynomials, is of this type [cf. (3)]'.

As a consequence of our condition we first see that the sequence

$$
\alpha_{0} \alpha_{n}, \alpha_{1} \alpha_{n-1}, \cdots, \alpha_{\rho} \alpha_{n-\rho}, \quad \rho=[n / 2],
$$

is positive, monotonically decreasing, and convex. Indeed Schwarz's inequality gives $\alpha_{k}^{2}<\alpha_{k-1} \alpha_{k+1}$, so that $\alpha_{k} / \alpha_{k-1}$ is increasing. The convexity follows from the representation

$$
\alpha_{k} \alpha_{n-k}=\int_{0}^{1} \int_{0}^{1} x^{k} y^{n-k} f(x) f(y) d x d y .
$$

We show further that

$$
\operatorname{sgn} f_{n}\left(\frac{\nu-\frac{1}{2}}{n} \pi\right)=(-1)^{\nu+1} \quad(\nu=1,2,3, \cdots, \rho=[n / 2])
$$

which is (in the case. of Legendre polynomials) equivalent to the lower estimate in the Markoff-Stieltjes theorem.*

To this end we use the following inequality due to Fejér [4]:

(11) $q_{1} \sin t+q_{2} \sin 2 t+\cdots+q_{m} \sin m t>\left\{\begin{array}{l}q_{m} / 2 \sin m t, \\ -q_{m} / 2 \sin (m+1) t,\end{array}, 0<t<\pi\right.$.

* We obtain immediately from (10) the existence of at least one zero in each of the intervals $\left(\nu-\frac{1}{2}\right) \pi / n<\theta<\left(\nu+\frac{1}{2}\right) \pi / n, \nu=1,2,3, \cdots, \rho-1$. An easy discussion shows then the existence of an additional zero in $\left(\rho-\frac{1}{2}\right) \pi / n<\theta<\pi / 2$. 
Here $q_{1}, q_{2}, \cdots, q_{m}$ is a positive, monotonically decreasing, and convex sequence. Fejér gives only the first inequality; the second arises from the first by considering the sequence $q_{1}, q_{2}, \cdots, q_{m}, q_{m}$, which has, of course, the same properties as $q_{1}, q_{2}, \cdots, q_{m}$.

Let $n$ be even. On putting $2 \theta=t, n / 2=m$, in the first inequality (11), we see at once that

$$
-\Im e^{-i n \theta} \sum_{k=0}^{[n / 2]}{ }^{\prime} \alpha_{k} \alpha_{n-k} e^{i(n-2 k) \theta}=\sum_{k=0}^{[n / 2]}{ }^{\prime} \alpha_{k} \alpha_{n-k} \sin 2 k \theta
$$

is positive in the interval $0<\theta<\pi / 2$. We have therefore

$$
f_{n}(\theta) \sin n \theta-g_{n}(\theta) \cos n \theta>0, \quad 0<\theta<\pi / 2,
$$

so that (10) is valid. If $n$ is odd, we put again $2 \theta=t,(n-1) / 2=m$, and observe that the particular value $t=(2 \nu-1) \pi / n$ satisfies the equation $m t$ $+(m+1) t=(2 \nu-1) \pi$, so that $\sin m t=\sin (m+1) t$. This means that the two expressions on the right side of (11) have opposite signs. The expression on the left side is therefore positive and this gives again (10).

\section{BIBLIOGRAPHY}

1. H. Bruns, Zur Theorie der Kugelfunktionen, Journal für Mathematik, vol. 90 (1881), pp. 322328.

2. L. Fejér, Sur les fonctions bornées et intégrables, Comptes Rendus, Paris, vol. 131 (1900), pp. 984-987.

3. L. Fejér, Abschätzungen für die Legendreschen und verwandten Polynome, Mathematische Zeitschrift, vol. 24 (1925), pp. 285-298.

4. L. Fejér, Einige Sätze, die sich auf das Vorzeichen einer ganzen rationalen Funktion beziehen; nebst A nwendungen dieser Sätze auf die Abschnitte und Abschnittsmiltelwerte von ebenen und räumlichen harmonischen Entwicklungen und von beschränkten Potenzreihen, Monatshefte für Mathematik und Physik, vol. 35 (1928), pp. 305-344.

5. E. Hille, Über die Nullstellen der Hermiteschen Polynome, Jahresbericht der Deutschen Mathematiker-Vereinigung, vol. 44 (1934), pp. 162-165.

6. A. Markoff, Sur les racines de certaines équations (seconde note), Mathematische Annalen, vol. 27 (1886), pp. 177-182.

7. G. P6lya, Über die Nullstellen gewisser ganzer Funktionen, Mathematische Zeitschrift, vol. 2 (1918), pp. 352-383.

8. T. J. Stieltjes, Sur les racines de l'équation $X_{n}=0$, Acta Mathematica, vol. 9 (1886), pp. 385400 .

9. Ch. Sturm, Sur les équations différentielles linéaires du second ordre, Journal de Mathématiques, vol. 1 (1836), pp. 106-186

10. G. Szegö, Bemerkungen zu einer Arbeit von Herrn Fejër über die Legendreschen Polynome, Mathematische Zeitschrift, vol. 25 (1926), pp. 172-187.

11. G. N. Watson, A Treatise on the Theory of Bessel Functions, Cambridge, University Press, 1922.

WASHINGTON UNIVERSITY,

St. Louis, Mo. 